\title{
OPEN: Optimized Path Planning Algorithm with Energy Efficiency and Extending Network - Lifetime in WSN
}

\author{
Syed Bilal Hussain Shah, Zhe Chen and Fuliang Yin \\ School of Information and Communication Engineering, Faculty of Electronic Information and Electrical Engineering, \\ Dalian University of Technology, Dalian, China
}

\begin{abstract}
In Wireless Sensors Networks (WSNs), researcher's primary focus is on energy preservation and prolonging network lifetime. More energy resources are required in case of remote applications of WSNs, where during active network stage some of the nodes die early, that shortens the network lifetime and decreases the stability of the network. It is mainly caused due to the non-optimal Cluster Heads (CHs) selection based on the single criterion and uneven distribution of energy. We propose a new distributed clustering protocol for both homogeneous and heterogeneous environments, named Optimized path planning algorithm with energy efficiency and extending network lifetime in WSN (OPEN). In the proposed protocol, we use timer value concept for efficient $\mathrm{CH}$ selection based on multiple parameters, e.g., residual energy, the average distance from its neighbors, and node density. Simulation results prove that OPEN performs better than the existing schemes regarding the network lifetime, throughput, and stability. The results explicitly explain the cluster head selection of OPEN protocol and efficient solution of uneven energy distribution problem.
\end{abstract}

ACM CCS (2012) Classification: Networks $\rightarrow$ Networks protocols $\rightarrow$ Networks protocol design

Networks $\rightarrow$ Networks protocols $\rightarrow$ Network layer protocols $\rightarrow$ Routing protocols

Networks $\rightarrow$ Networks types $\rightarrow$ Ad hoc networks $\rightarrow$ Moble ad hoc networks

Networks $\rightarrow$ Network performance evaluation $\rightarrow$ Network simulations

Keywords: path planning algorithm, sleep, awake, routing, network lifetimes

\section{Introduction}

Wireless Sensor Network (WSN) is a combination of many small sensors having low en- ergy resources. WSNs have rich applications in many fields, such as environmental sciences, natural disaster monitoring, biosciences and traffic control systems, etc. Sensor nodes deployed in the field can't be recharged to retain their energy resources; hence energy efficiency is a major issue in WSNs.

Efficient energy utilization requires designing intelligent algorithms. Among so many routing path planning algorithms, clustering is the most useful energy efficient technique. Clustering divides the whole network into small groups of sensors called clusters. For each cluster, a leading node called Cluster Head $(\mathrm{CH})$ is selected [1]- [3]. Selection of the $\mathrm{CH}$ is based on some particular criteria. Other members of the cluster are called cluster members or normal nodes [4]. These member nodes sense the environment and assemble the appropriate data periodically, after which they send this data to the $\mathrm{CH}$. On the reception of data from cluster members, $\mathrm{CH}$ aggregates the data and forwards it to the central server called Base Station (BS) [5].

As $\mathrm{CH}$ has more responsibilities like data collection, data aggregation and transmission to the $\mathrm{BS}$, so it consumes more energy as compared to the member nodes. That's the reason why $\mathrm{CHs}$ are more volatile to die earlier [6]. Better $\mathrm{CH}$ selection in each cluster of the whole network can balance the energy consumption, which can improve network's lifetime and stability. Energy consumption of the $\mathrm{CH}$ also depends on the distance from the BS. $\mathrm{CH}$ at the longer distance from the BS uses more energy to forward 
their data to the BS as compared to the nodes closer to the BS. In multi-hop communication, $\mathrm{CHs}$ away from the $\mathrm{BS}$ forward their data via the $\mathrm{CHs}$, that work as an intermediary, to the BS [6]. In this scenario, energy consumption load on the nodes closer to the BS is greater. This energy imbalance issue is called hot spot problem [7]-[9]. CH selection based on the single parameter of nodes may create some problems. For example, if $\mathrm{CHs}$ are selected based on residual energy or node's distance from the BS, then the nodes closer to the BS become $\mathrm{CHs}$ in single-hop communication. Moreover, WSNs deployment takes place mostly in the remote areas, which are inaccessible and composed of a large number of sensor nodes. In such network model, the centralized algorithms where BS makes the decisions are impracticable and may not be the optimal choice.

In this paper, a distributed routing path planning algorithm is proposed, where nodes are responsible for the $\mathrm{CH}$ selection. We consider multiple parameters of nodes to select the most suitable $\mathrm{CH}$. Every $\mathrm{CH}$ with different cluster radius creates a cluster with uneven size regarding the number of nodes. The $\mathrm{CH}$ close to the $\mathrm{BS}$ have lesser number of nodes. Hence, the smaller size of the cluster allows the CHs to preserve energy. This energy is employed for forwarding the data of CHs that are far from the BS. In this way, the efficient solution of hot spot problem is suggested which extends the lifetime of the network.

The paper is organized as follows: Section two contains literature review and the work related to our proposed scheme. The third section explains the network design model. The fourth part highlights the proposed algorithm with the observations and experiments that show the comparison of the proposed scheme OPEN with the state of the art routing protocols. The fifth section shows complete results discussion, while the sixth section contains the conclusion and future work that can be beneficent for the future research.

\section{Literature Review}

Younis et al. present a detailed survey of different types of clustering techniques in WSNs and identify two major problems in these methods
[10]. The first issue is related to the parameters that are used to select $\mathrm{CH}$ and the second one to the nature of clustering protocols which are either probabilistic or iterative. Deosarkar et al. explain the basic rules for the selection of $\mathrm{CH}$ in WSNs [11]. They classify the $\mathrm{CH}$ selection methods into three categories. First, the deterministic scheme in which node degree or node ID (fixed parameters) is used to determine the rules to select the $\mathrm{CH}$. The second method to decide the $\mathrm{CH}$ is the adaptive scheme which uses variable information of the sensor or its surroundings. The last category is the combined plan, combining deterministic and adaptive schemes, which use both fixed and variable parameters for the selection of $\mathrm{CH}$ within a cluster.

In this paper, we discuss an underlying clustering protocol called (LEACH) Low Energy Adaptive Clustering Hierarchy [1]. Operations of this protocol are divided into multiple rounds. Each round in LEACH has two phases; setup phase and steady state phase. In the setup phase, all sensor nodes organize themselves into clusters. However, normal nodes send data to their respective $\mathrm{CHs}$ in the steady state phase. Each normal node elects itself as a $\mathrm{CH}$ in the setup phase by generating a random value between $(0-1)$. If the selected random number is lesser than the threshold, then the node becomes the $\mathrm{CH}$ for that particular round. The threshold is given in equation (1).

$$
T_{h}= \begin{cases}\frac{P_{r}}{1-P_{r} \cdot\left(\left(C_{r}\right) \bmod \frac{1}{P_{r}}\right),}, & \text { if } n \in G_{n} \\ 0, & \text { otherwise }\end{cases}
$$

where $P_{r}$ represents the required percentage of the $\mathrm{CH}, G_{n}$ is the number of nodes that have not been elected as $\mathrm{CHs}$ during the last $1 / p_{r}$ rounds and $C_{r}$ is the particular round number.

After the selection, these CHs advertise themselves as $\mathrm{CHs}$ to all member nodes. On reception of the advertisement, normal nodes determine the cluster to join based on the distance from the CHs. After the cluster formation, normal nodes send their data to the relevant $\mathrm{CHs}$. After the receipt of the data from the normal nodes, $\mathrm{CHs}$ compress, aggregate and ultimately forward the data to the BS. 
Heinzelman et al., designed an advanced version of LEACH called Centralized LEACH(CLEACH) [12]. This protocol uses the location tracking such as GPS for optimal formation of clustering and BS is responsible for cluster formation here. In the setup phase, each node broadcasts a packet including residual energy and its location to the BS. Then the BS selects $\mathrm{CHs}$ based on the information received from the nodes by applying a Simulated Annealing (SA) algorithm. The steady state phase of C-LEACH is the same as LEACH. Network lifetime and stability can be increased significantly due to optimal cluster formation. Based on the central concept of LEACH, many routing protocols have been proposed, such as protocol based on multi-hop communication is LEACH-M [13]. Multi-Level Clustering Algorithm is EEMLC [14], and, similarly, schemes based on heterogeneous networks are EEHC [15], DEEC [16] and CEEC [6].

Liu et al. projected a novel distributed clustering protocol named Energy-Aware Data Gathering (EADEEG) protocol for WSN [17]. In this plan, selection of $\mathrm{CH}$ is based on the ratio between the residual energy of a particular node and the average residual energy of its neighbor nodes, which improves network stability and prolongs network lifetime. This protocol is a competent energy efficient protocol; however, it still has some limitations. It chooses " $2 r c$ " inter-cluster communication radius, which does not always ensure connectivity among CHs. Another problem in this scheme is the presence of isolated points in some cases. Zhou et al. proposed a new distributed energy aware clustering protocol called BPEC for the efficient solution to these problems [18]. This protocol uses two criteria for the selection of $\mathrm{CH}$. First and the primary criterion is the same as EADEEG, which is the ratio between the residual energy of a particular node and the average residual energy of its neighbor nodes. Another criterion is the node's degree. This protocol overcomes the "isolate point" problem in EADEEG and ensures the connectivity among all CHs. There were also some other energy efficient protocols which eliminate "isolate point" problem [19] - [23]. In these protocols, CHs are nominated iteratively.

All the above-discussed protocols have solutions for the "isolate point" problem. However, they do not provide a solution for the Energy
Hole problem. Soro et al. suggested a unique clustering protocol which overcomes this issue [24]. Here, the network is divided into circles. Each circle consists of clusters of the same size. However, clusters in different circles may vary in size. Energy consumption is balanced by deploying the nodes with high energy as CHs. Positions of $\mathrm{CHs}$ must be calculated in advance so that some high energy nodes are chosen as CHs. This protocol can prolong network lifetime more efficiently.

Li et al. propose an unbalanced clustering protocol [25]. CH selection in this protocol is based on the residual energy of nodes. Initially, each node temporarily becomes the $\mathrm{CH}$ with probability $\mathrm{P}$. Then, these temporarily selected CHs build random (not fixed) size clusters using rough competition ranges. The clusters away from the BS have a larger size as compared to the clusters closer to the BS. So, the clusters near the BS can save some amount of energy for multi-hopping to forward the data of multiple clusters to the BS. This balances the energy utilization. In this protocol, cluster generation quality depends on the used probability $\mathrm{P}$. Therefore, in some cases of $\mathrm{P}$, the "isolate point" problem remains, as shown in Figure 1. The major focus of this paper is the need for a technique to determine the $\mathrm{CH}$ in such a way that the energy is equally distributed on each cluster member in every round, which automatically improves stability and lifetime of the network.

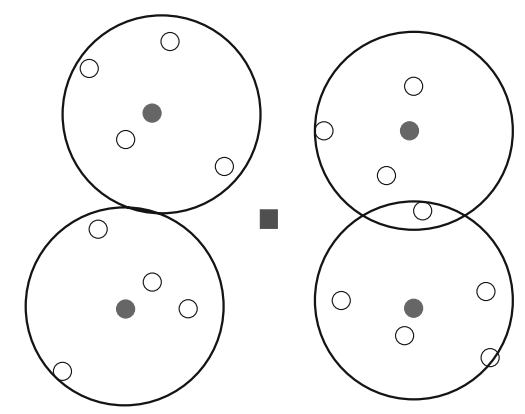

Figure 1. Advanced Network Clustering Mode.

\section{Network Design of the Proposed Protocol}

We use a distributed scheme for energy balancing to conserve energy. In the scheme, ev- 
ery single node is responsible for making decisions related to the selection of $\mathrm{CH}$ for each round. In every round, $\mathrm{CHs}$ collect data from their respective cluster members and aggregate that data before forwarding it to the BS. The purpose of the proposed model is to design a clustering path planning algorithm which can provide better lifetime and stability to the sensor network.

The lifetime of the WSN network is defined as a period of time until which a section of the sensor nodes dies due to energy deficiency. In WSN, sensed updates from the sensor nodes are continuously sent to the BS. It is considered as one round, which is a synchronized periodic re-clustering operation. It takes part in getting information from all of the sensor nodes and sending it to the BS until the first node dies in the sensor network. Coverage is also used as a parameter of the lifetime, which is defined as time period until the coverage of network is reduced to a specified percentage of the area.

\subsection{Radio Model}

In the paper, we use the first order radio model [1]. In this model, a sensor node consists of seven components including, transmission and reception antenna, transmission and reception electronic devices, transmission amplifier, sensor and data processor, as shown in Figure 2. The energy of the system is utilized in three operations: data transmission, data reception, and data compression. System energy equation is as follows:

$$
E_{T x}=E_{\text {elect }} \times P_{k}+E_{a} \times P_{k} \times D^{2}
$$

where $E_{T x}$ is the energy consumed for transmission per bit, $E_{\text {elect }}$ is the amount of circuit energy, $E_{a}$ is the amount of energy consumed in the amplifying process, $P_{k}$ is the number of transmitted bits, $D$ is the distance between a node and its respective $\mathrm{CH}$ or between $\mathrm{CH}$ and BS.

At the receipt, energy is consumed due to receiving data bits and receiver electronics.

$$
E_{R x}=\left(E_{\text {elect }} \times P_{k}\right)(n) A_{e}=E_{A D} \times P_{k} \times n
$$

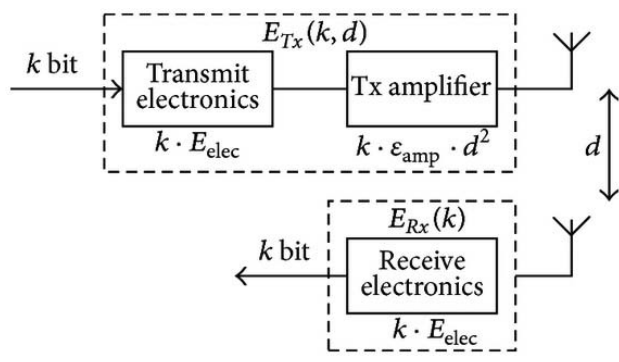

Figure 2. Network topology of the proposed scheme.

The third source of energy consumption in the sensor networks is data aggregation done by the $\mathrm{CH}$. In data collection, redundancy of similar data packets is eliminated, and data packets are merged so that the number of transmissions to the BS is reduced. The equation shows data aggregation energy $\left(E_{A D}\right)$ as:

$$
A_{e}=E_{A D} \times P_{k} \times n
$$

where $n$, represents the total number of nodes in the network.

\subsection{Assumptions}

In the proposed model, we make some assumptions to make it simpler. They are:

- Each data packet has a unique data packet number which is used to identify the source of that data packet.

- BS is fixed.

- All the sensor nodes are homogeneous, which means that they have same initial energy.

- The size of the data packet is constant. So, it sends the same amount of data in every round.

- Working of a cluster of sensor nodes is synchronized to the $\mathrm{CH}$, and each sensor node can communicate directly with its $\mathrm{CH}$.

- The transmission energy of the sensor nodes depends on the distance from $\mathrm{CH}$ or its next hop (in the case of multi-hopping).

- The position of the sensor nodes is fixed. Hence, the distance between the sensors has to be calculated once using GPS or any other non-GPS technique. 
- Ideal transmission conditions are used. It means that there is no loss or delay of data packets during transmission.

- CHs perform transmissions to the BS and data aggregation.

We can summarize these assumptions as fixed homogeneous sensor nodes continuously transmit the same amount of aggregated data to the stationary single BS. The first order radio model is the simplified model which fits in the real life systems. Parameters of this radio model can be changed according to the system requirements.

\section{Proposed Model (OPEN)}

As stated earlier, our key focus is on the extension and improvement of the stability and lifetime of the network. So the network lifetime optimization can be performed through energy balancing. In this section, we propose a scheme which resolves the stability and lifetime issues of the network.

In the scheme, distributed clustering techniques are used for homogeneous and static nodes. The decision of $\mathrm{CH}$ selection is made solely by the nodes based on their local information. The timer value concept is used to select the most suitable node as $\mathrm{CH}$. Each node executes an algorithm individually and generates a value called timer value $T_{v}$. The Timer value $T_{v}$ depends on three parameters of a node that are:

- Residual energy

- Node's density

- Average distance from its neighbor

A node with the lesser timer value is selected as $\mathrm{CH}$ which communicates with the $\mathrm{BS}$ and forwards the data to other member nodes in its cluster. $\mathrm{CH}$ consumes more energy than the other nodes in its cluster. Therefore, if the selected $\mathrm{CH}$ remains constant throughout all the rounds, then it dies before the other nodes and decreases the stability of the network. So, to overcome this problem, the responsibility of a node as a $\mathrm{CH}$ switches among the nodes by triggering the $\mathrm{CH}$ selection process periodically. Hence, the overall process of our protocol consists of synchronized and periodic rounds. Each round is divided into two phases: cluster organization phase and cluster communication phase. In cluster organization phase, the $\mathrm{CH}$ is selected, and this process is repeated periodi- cally after every five rounds. However, the data is sent to the BS during cluster communication phase. A comprehensive description of the two phases is given in the following subsections.

\subsection{Cluster Organization Phase}

In this phase, nodes link together to form clusters and select optimal $\mathrm{CHs}$ for their respective clusters. Cluster organization phase is further divided into three phases: neighbor information collection phase, cluster head election phase, and node association phase. All the aspects are discussed in the following subsections.

\subsubsection{Neighbor Information Collection Phase}

In this phase, node's neighbor information collection is carried out through a technique called node localization. Every node discovers its surroundings by acting as a beacon. Initially, each node broadcasts a beacon message in its radio range. After broadcasting the beacon messages, nodes observe the medium for similar beacon messages. Every node which receives the broadcasted beacon message replies with an acknowledgment message. Each node then calculates the distance from its neighbor through some non-GPS technique like RSSI [26] or the time difference between the arrivals of the ultrasound pulses. The nodes use Carrier Sense Multiple Access (CSMA) MAC protocol to avoid collision between any two beacon messages [27]. The nodes keep a record of the distance information of all their neighbors in their neighbor table. The neighbor table contains information about the number of neighbors and the distance of each neighbor. Neighbor information collection phase is repeated after a particular time period to reduce the beacon overhead, which decreases energy consumption. As the nodes are static (that is, their location remains fixed), nodes repeat the neighbor information collection phase after every ten rounds to highlight the dead neighbors. The nodes use this information for $\mathrm{CH}$ election process which is explicitly explained in the subsection.

\subsubsection{Cluster Head Election Phase}

As soon as the neighbor information collection phase is over, nodes are ready to elect themselves as $\mathrm{CH}$. Each node calculates the aver- 
age distance from its neighbor using the information in the neighbor table by the following equations:

$$
\begin{gathered}
D_{t}=\sum_{i=1}^{n} d i s t_{i} \\
D_{a}=\frac{D_{t}}{N}
\end{gathered}
$$

where dist $_{i}$ is the distance of neighbor "i", $D_{t}$ is the sum of all the nodes' distances, $N$ is the total number of neighbors and $D_{a}$ is the average distance of the node from its neighbors.

After calculating the average distance, each node calculates timer value $T_{v}$. Based on this value each node makes the decision whether to be the $\mathrm{CH}$ for a particular round or not. $T_{v}$ can be calculated from the following equation:

$$
T_{v}=\frac{D_{a}}{N_{n} \times E_{r}} .
$$

Assigning weights

$T_{v}=\frac{D_{a} \times W_{d a}}{\left(N_{n} \times W_{n}\right) \times\left(E_{r} \times W_{e}\right)}=\frac{D_{a}}{\left(N_{n} * E_{r}\right)} *\left(W_{t} o_{t}\right)$,

where

$$
\left(W_{t} o_{t}\right)=\frac{W_{d a}}{W_{n} \times W_{e}}
$$

where the total number of neighbors of a node is $N_{n}, E_{r}$ is the residual energy of the nodes, while, $W_{n}$ and $W_{e}$ are the weights of each criterion with the value between 0 and 1. These weights are assigned to each criterion which normalizes each criterion value and shows the relative importance of each criterion. Every criterion has its importance in the $\mathrm{CH}$ selection phase. The value of each criterion weight lies between 0 and 1 and the cumulative value is 1 . We use weights to give values to the equation between $0-1$ and see the effect of three parameters on $T_{v}$, that is ultimately used to select the efficient $\mathrm{CH}$.

For example, as we assign a maximum weight value to $N_{n}$, the node with the highest number of neighbors has a better chance to become the $\mathrm{CH}$. However, other criteria will also be considered for that node. While calculating the timer value for every node, $N_{n}$ and $E_{r}$ are inversely proportional to $T_{v}$, but the distance is directly proportional. This means that $\mathrm{CH}$ will be a node with shortest $T_{v}$, a node with the shortest distance to its neighbors, highest residual energy and number of neighbors, as clearly shown in the equation 7 . All the nodes set their timers according to the $T_{v}$ value and start executing them. A node with the lesser timer value $T_{v}$ is selected as $\mathrm{CH}$. If the timer of a specific node completes its calculation and it does not receive any cluster head broadcast message from the other member nodes, the node selects itself as a $\mathrm{CH}$ and advertises itself by a broadcast message within its cluster radius range $C_{r}$. The $C_{r}$ of all $\mathrm{CHs}$ is not equal and can be calculated by the following equation:

$$
C_{r}=\left[1-w \frac{d i s t_{\max }-d i s t_{i}}{d i s t_{\max }-d i s t_{\min }}\right] R_{\max }
$$

where dist $_{\max }$ and dist $_{\min }$ are the maximum and minimum distances of a node from the BS in the network, dist $t_{i}$ is the distance of a node $i$ from the $\mathrm{BS}, w$ is the weight factor and its value is in the range of $[0,1]$ and $R_{\max }$ is the maximum value of the competition cluster radius. $R_{\max }$ can be calculated by the following equation:

$$
R_{\max }=\frac{E_{c r}}{E_{t}}
$$

where $E_{c r}$ is the energy consumption of a single round and $E_{t}$ is the total initial energy of the network.

As shown in equation (7), $T_{v}$ depends on three parameters of a node: residual energy, the number of neighbors and the average distance of a node from its neighbors. $T_{v}$ value increases as the node's distance from its neighbors increases. However, $T_{v}$ is also directly proportional to the node's average distance from its neighbors. A node with the greater distance from its neighbors consumes the higher energy in communication with its $\mathrm{CH}$ within a cluster. So to conserve energy, the best $\mathrm{CH}$ will be the node with minimum distance from its neighbors, highest residual energy and number of neighbors. Furthermore, $T_{v}$ is inversely proportional to the residual energy and number of neighbors.

The most suitable node to act as $\mathrm{CH}$ is the one with higher residual energy and the greater number of neighbors. 


\subsubsection{Node Association Phase}

In the node association phase, non- $\mathrm{CH}$ nodes send a request message to the $\mathrm{CH}$ in each cluster. The $\mathrm{CH}$ of each cluster creates TDMA schedule for the data transmission between the non-cluster head nodes and itself. $\mathrm{CH}$ sends TDMA schedule in the acknowledgment (ACK) message to the non-cluster head nodes of the cluster. Each non-cluster head node receives this ACK message and associates itself with the relevant $\mathrm{CH}$.

Proposed model utilizes S-MACS, a protocol specially designed for WSNs that circumvents the problems of idle listening, collision, and overhearing. However, it still keeps the flexibility of a contention-based scheme. Each node changes between the sleep and listening mode periodically. The duration of these periods is determined by a schedule that is negotiated among the neighboring nodes during a short schedule synchronization phase between each sleep and listening cycles. Because of S-MACS medium access technique utilized by our proposed model, the sensor nodes save the energy resources, which results in the better network lifetime and stability of proposed model.

\subsection{Cluster Communication Phase}

In cluster communication phase, nodes in each cluster route the sensed data to the BS. The $\mathrm{CH}$ node performs the role of the router in the network. All the associated nodes transmit their data to the $\mathrm{CH}$ in the scheduled TDMA slots, which further route the data to the $\mathrm{BS}$. $\mathrm{CH}$ receives the sensed data from the normal nodes, aggregates it and sends it to the $\mathrm{BS}$. The $\mathrm{CH}$ nodes can directly transmit data to the BS.

\subsubsection{Nodes Communicate in Scheduled TDMA Fashion.}

In each cluster, all duties are performed in $(\Delta \mathrm{T})$ time intervals which are dynamically changed [28]. There are three kinds of nodes in a cluster: $\mathrm{CH}$, which gather data from all the member nodes in a cluster, active nodes which are nodes that have data ready for sending, and passive nodes which presently have no data for sending.

Channel-1 will act as a control channel for request messages from sensor nodes to $\mathrm{CH}$, allo- cated to more than one sensor nodes in a cluster. One remaining channel from $\mathrm{N}$ channels of cluster head will be used as channel-R, that is shared between all the nodes in a cluster.

The operations of a cluster on $\Delta \mathrm{T}$ are organized in three sequential phases: request phase, scheduling phase, and data transmission phase.

Request phase. At the beginning of each $\Delta \mathrm{T}$, the network layer determines the active nodes, which start request phase on Channel-1 with $\mathrm{CH}$. As Channel-1 will be allocated to more than one active nodes, hence the adaptive contention window protocol [29] can be used to avoid a collision and achieve better performance on this channel. When active nodes receive acknowledgment of its REQ message, they go to standby mode and wait for scheduling message from $\mathrm{CH}$.

Scheduling phase. Each $\mathrm{CH}$ computes a suitable schedule, based on the priority and other QoS requirements specified in REQ message. Then cluster heads broadcast scheduling messages through N-1 channels to maintain synchronization. $\mathrm{CH}$ assigns a channel as channel-2 to each active node for data transmission. If the number of active nodes in a cluster is more than $\mathrm{N}-1$, a time slot in a channel is allocated to more than one active nodes on channel-2 to avoid collisions. Consequently, the scheduling message contains a channel and a time slot in it as channel-2 for each active node.

Moreover, new $\Delta \mathrm{T}$ that is calculated based on REQs is included in the scheduling message. New $\Delta T$ specifies the end of this interval and start of the next interval. A cluster time intervals have been illustrated in Figure 6 for three intervals.

\subsection{Transmission Phase}

After receiving a scheduling message, an active node could send its data the assigned channel. If a time slot in a channel is assigned to an active node, it can go to sleep mode until its time slot approaches. Some passive nodes may get active in this phase, can send its REQ messages at channel-R. So, if free channels or time slots in channels have been assigned to such nodes, they could send their data at a scheduled time.

CDMA can be used to avoid the inter-cluster collision, each cluster uses a different set 
of CDMA codes to minimize the interference between clusters. More data per bit need to be transmitted, as it allows multiple transmission using the same frequency. To reduce interference between neighboring clusters, a different spreading code is used in each cluster. In free slots, gateway nodes pseudo-randomly re-tune (on a slot by slot basis) their receivers to the codes of the adjacent clusters to maintain synchronization with all neighbors.

However, under the cramped and harsh conditions, the connection between the CHs may drop, and the data might be lost. Furthermore, the energy consumption in the transmission increases as the distance between $\mathrm{CHs}$ and the $\mathrm{BS}$ increases. For the efficient solution of this problem, CHs communicate with the $\mathrm{BS}$ in a multi-hop tree like manner while the BS acts as the root. In this multi-hop tree, the nodes near the $\mathrm{BS}$ in a particular range $R_{b s}$ directly communicate with the BS. However, the $\mathrm{CH}$ nodes, which are out of the range $R_{b s}$, forward the aggregated sensed data to the next nearest $\mathrm{CH}$ in the direction of the BS. The main advantage of the multi-hop tree like communication $(\mathrm{CH}$ to $\mathrm{CH}$ ) is that it minimizes the energy consumption in the data transmission, increasing the network lifetime and stability. In addition, it also minimizes the data loss and increases the throughput. The overall flow diagram of the $\mathrm{CH}$ selection phase is given in Figure 3.

\section{Simulation and Results Discussion}

In this section, performance of the proposed scheme is compared with LEACH, LEACH-C, DEEC and also with some known state of the art protocols like PEGASIS, CEEC, MLEACH and SEP using MATLAB as the simulation tool. The reason why these protocols are compared with the proposed protocol is to highlight and indicate that the issues found in them are explicitly resolved in our proposed scheme. Figure 1 shows the advance network clustering model which specifies the position of clusters, $\mathrm{CHs}$ and BS deployed in the particular area.

In the simulation setup, sensor nodes are randomly dispersed in a field of the area with the $\mathrm{BS}$ at the top of the field to make a comparison among LEACH, LEACH-C and DEEC [1], [12], [16]. In the simulations setting, nodes

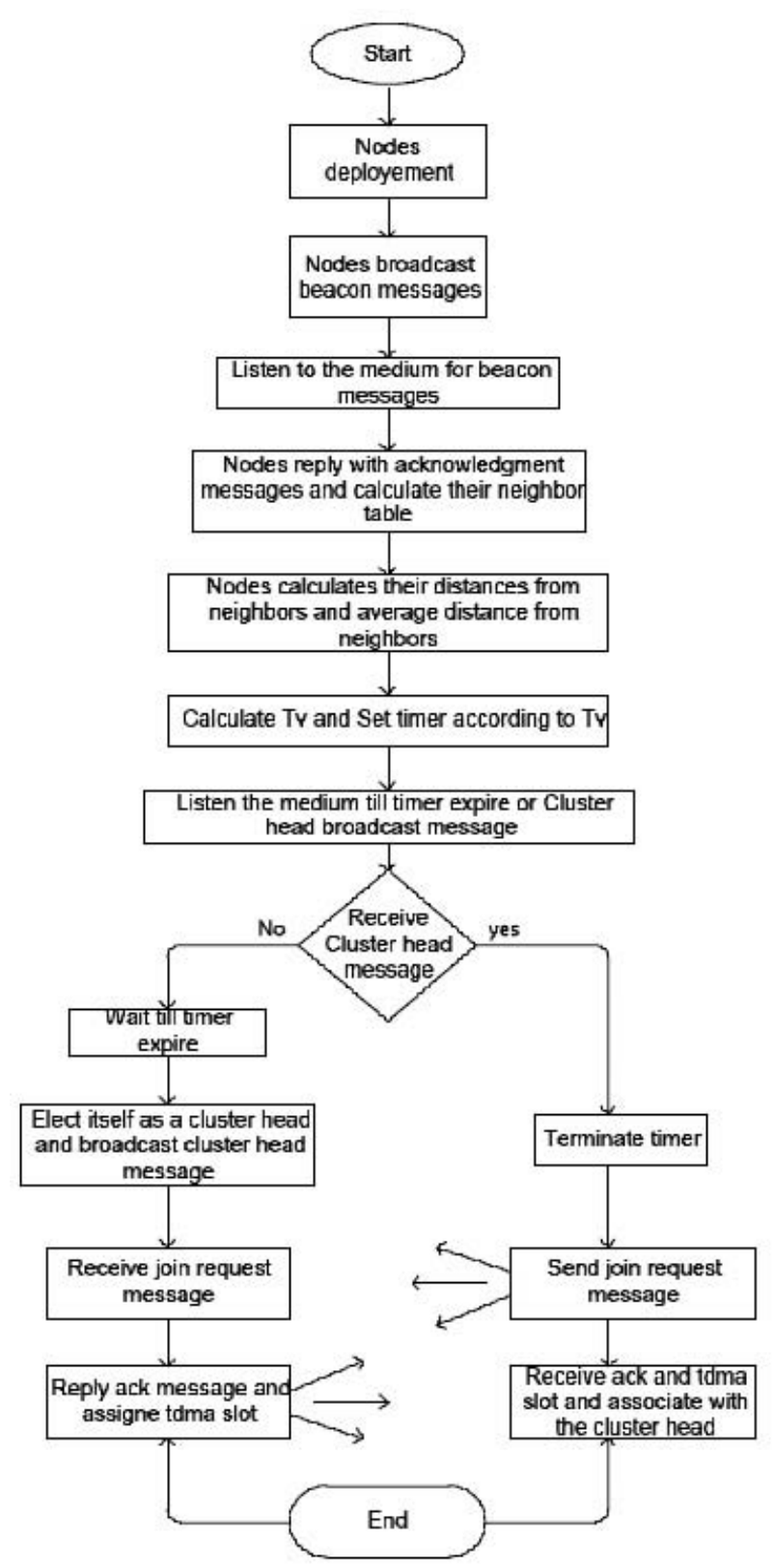

Figure 3. Flow diagram of the proposed scheme for $\mathrm{CH}$ selection.

with the initial energy, transmit fixed size data in every round. Other simulation parameters are given in Table 1.

Simulation scenario of the proposed model is elaborated in Figure 4, in which random node's deployment is visible where nodes are scattered without any localization plan. $\mathrm{CHs}$ inter-cluster communication with the $\mathrm{BS}$ and the communication of the associated nodes with the $\mathrm{CHs}$ during intra-cluster communication can also be seen in Figure 4. 
Table 1. Simulation parameters.

\begin{tabular}{|c||c|c|}
\hline Parameter & Description & Value \\
\hline \hline Eo & $\begin{array}{c}\text { Initial energy of } \\
\text { sensor nodes }\end{array}$ & $100 \mathrm{~m} \times 100 \mathrm{~m}$ \\
\cline { 2 - 3 } $\mathrm{AE}$ & $\begin{array}{c}\text { Energy consumption } \\
\text { on data aggregation }\end{array}$ & $50 \mathrm{pj} / \mathrm{bit} \mathrm{j}$ \\
\hline $\mathrm{n}$ & Number of nodes & 100,200 \\
\hline $\mathrm{Pk}$ & Packet size & $4000 \mathrm{bit}$ \\
\hline \multirow{2}{*}{$\mathrm{ETx}$} & $\begin{array}{c}\text { Energy consumption } \\
\text { per bit by } \\
\text { transmission circuitry }\end{array}$ & $50 \mathrm{~nJ} / \mathrm{bit}$ \\
\hline ERx & $\begin{array}{c}\text { Energy consumption } \\
\text { per bit by receiver } \\
\text { circuitry }\end{array}$ & $50 \mathrm{~nJ} / \mathrm{bit}^{2}$ \\
\cline { 2 - 3 } Eamp & $\begin{array}{c}\text { Energy consumption } \\
\text { on amplification }\end{array}$ & $100 \mathrm{pJ} / \mathrm{bit} / \mathrm{m}^{2}$ \\
\hline
\end{tabular}

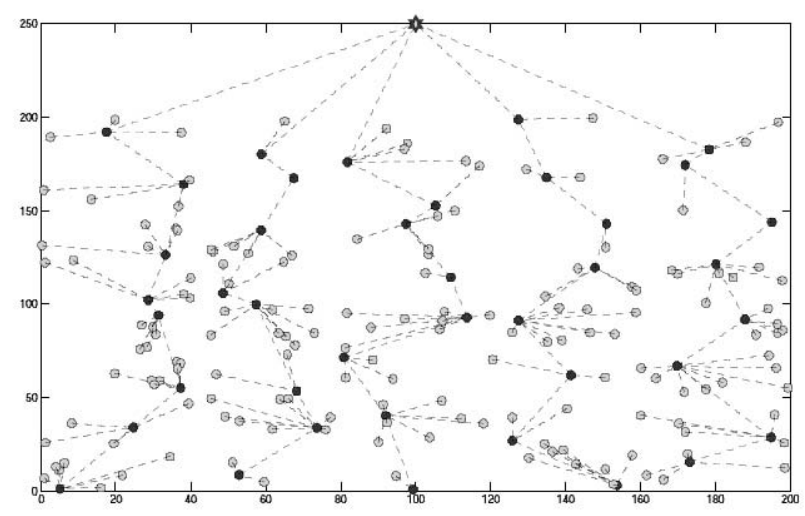

Figure 4. CHs and BS communication layout of the proposed scheme.

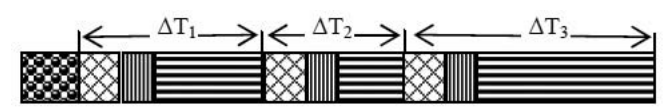

Channel-1 assignment phase
Request phase $(\Delta \mathrm{Tr})$
Scheduling phase (chanel-2 assignment phase)
Data transmission phase

Figure 6. Operations within a cluster.

In simulation experiments, the network lifetime of the proposed scheme and existing protocols are shown in Figure 5. It depicts that the overall lifetime of our proposed scheme is 2325 rounds. However, the lifetime of LEACH, LEACH-C, and DEEC are 1442, 1867 and 1643 rounds respectively.

The proposed plan increases lifetime of the network by almost $140 \%$. The CHs selection on multiple parameters and the multi-hop tree like communication play a fundamental role in improving the lifetime. However, other protocols consider only residual energy or some probability $\mathrm{P}$ for $\mathrm{CHs}$ selection. Figure 5 shows that performance of the proposed scheme is better than all the other protocols, including LEACH-C which is based on the centralized algorithm. However, our proposed scheme has distributed property. So, it can be concluded that the proposed protocol performs better in small-scale as well as in large-scale networks. The $\mathrm{CHs}$ responsibilities are properly divided between the nodes and $\mathrm{CHs}$ are selected in every round based on the timer value concept, which increases stability of the network.

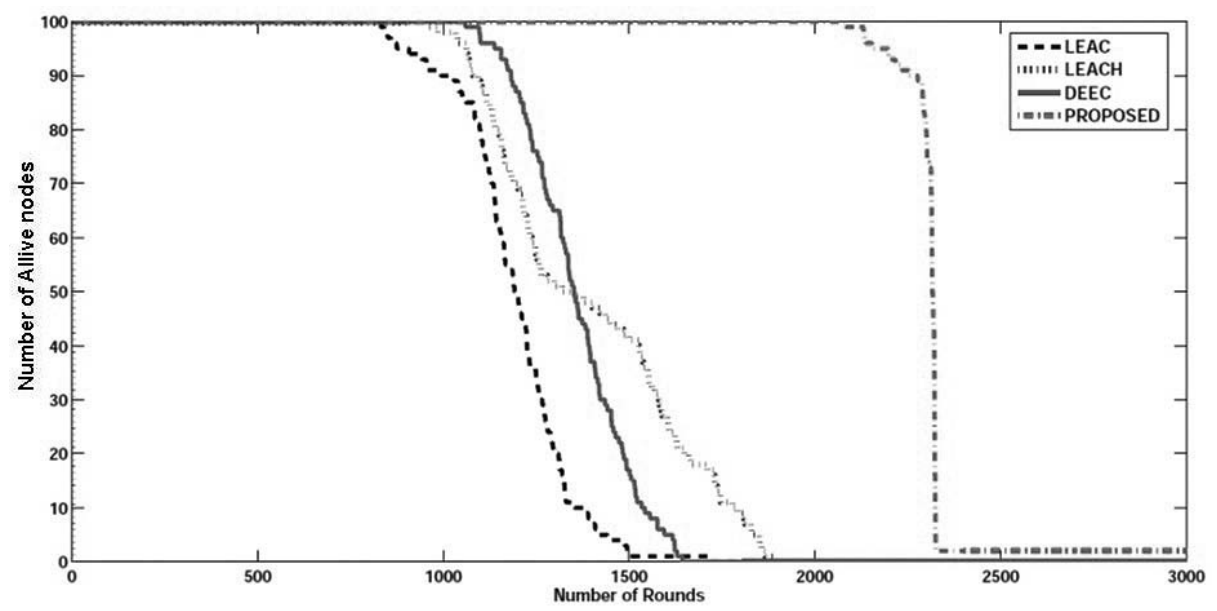

Figure 5. Network lifetimes comparison of LEACH, LEACH-C, DEEC and the proposed scheme. 


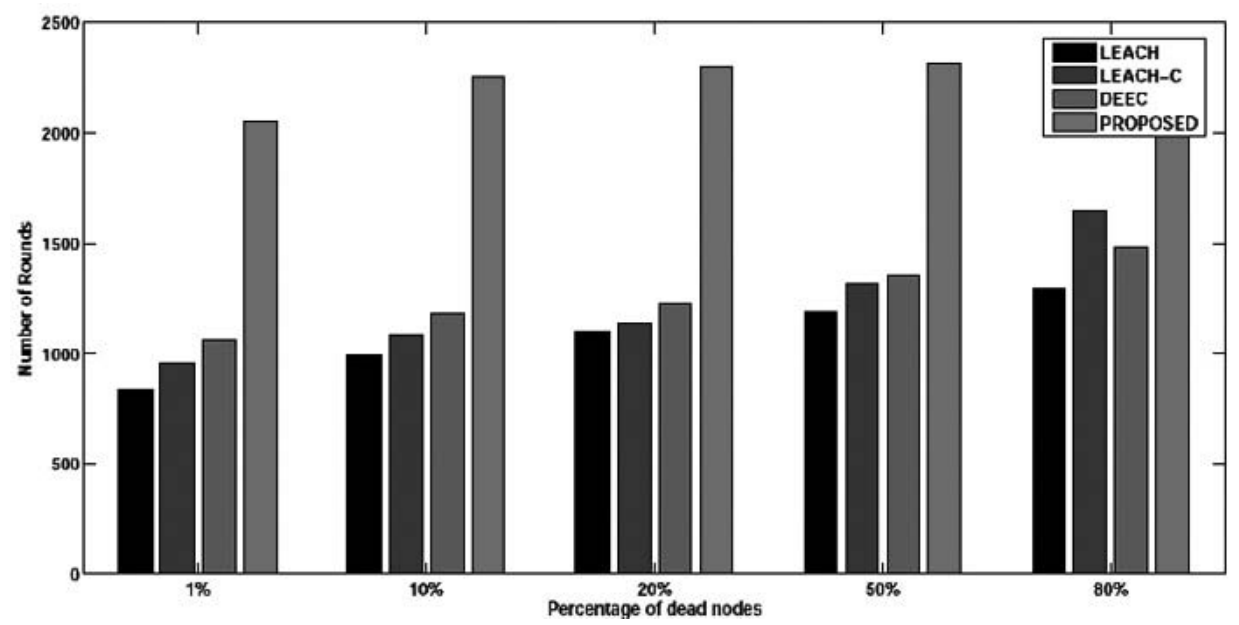

Figure 7. The percentage of dead nodes in LEACH, LEACH-C, DEEC and the proposed scheme.

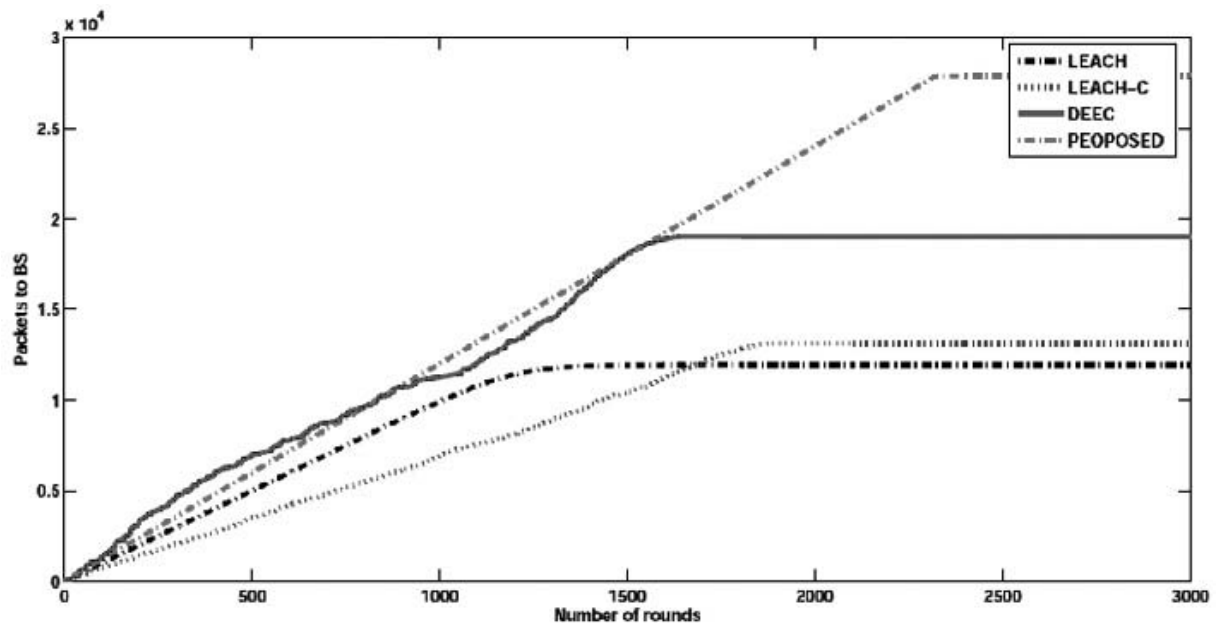

Figure 8. The throughput of the network for LEACH, LEACH-C, DEEC and proposed scheme.

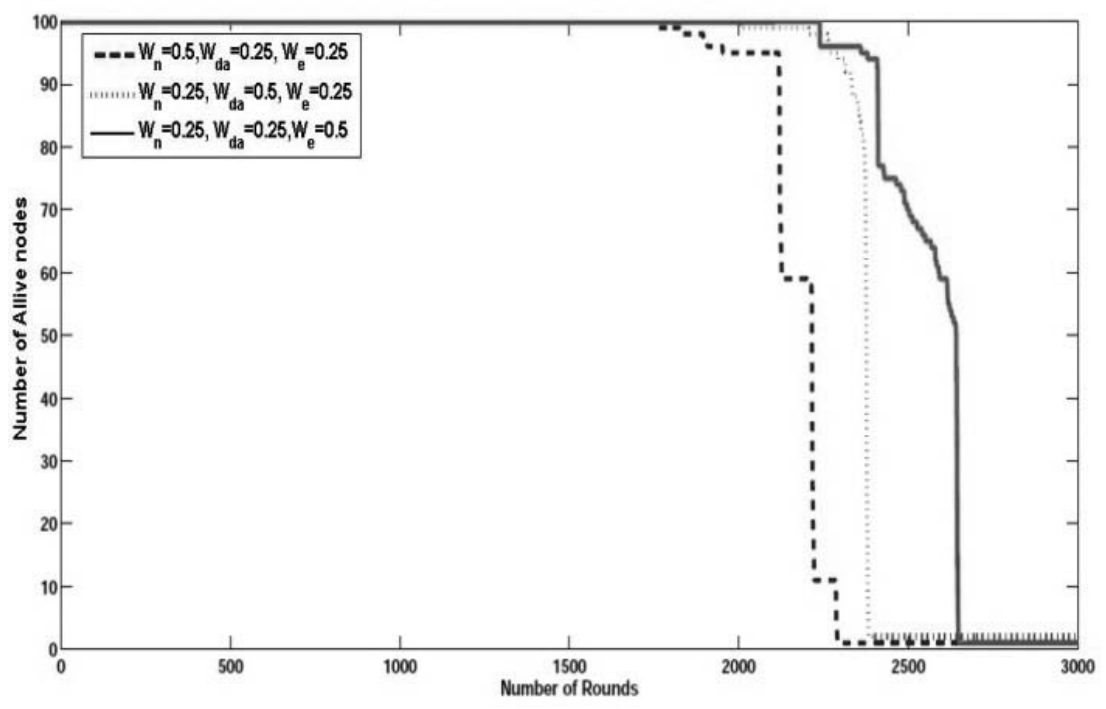

Figure 9. Different weights mapped to each criterion. 


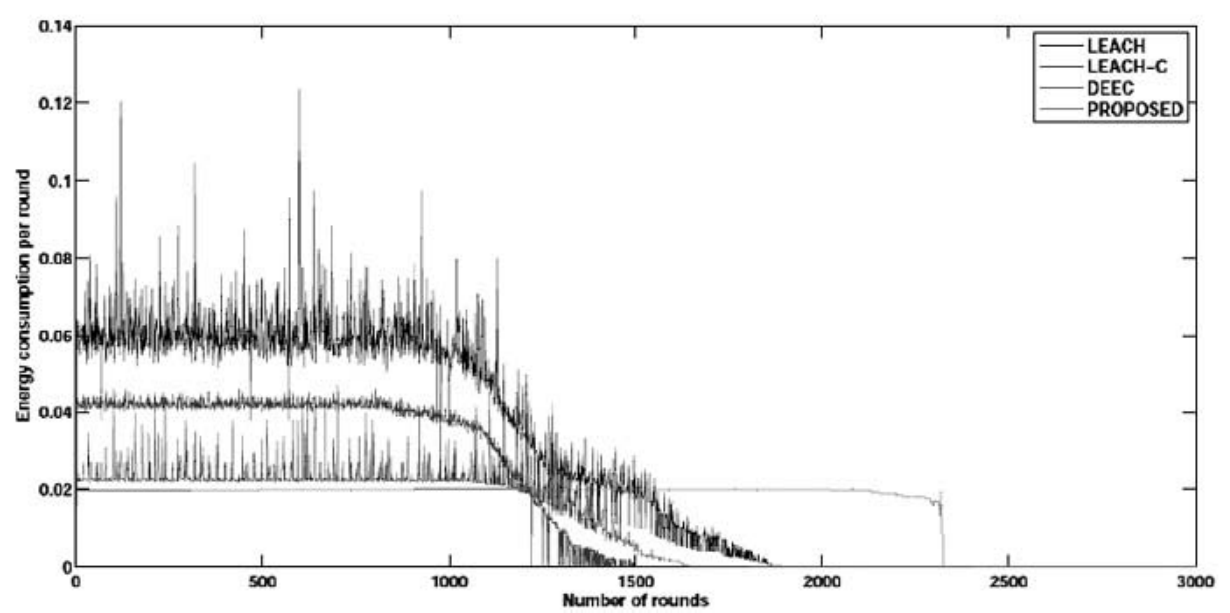

Figure 10. Energy consumption per round for LEACH, LEACH-C, DEEC and proposed scheme.

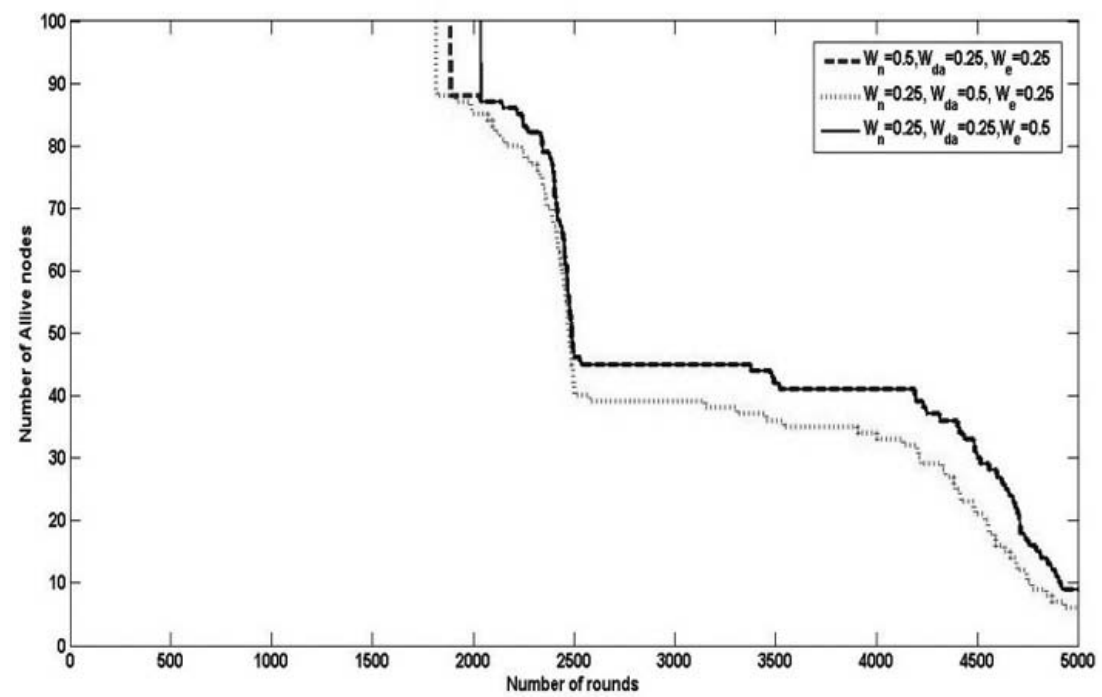

Figure 11. Different scenarios with different weights assigned to each criterion to determine network lifetime.

Figure 7 shows that $1 \%$ percent of the nodes die in the proposed scheme in 2000 rounds and 80 percent of the nodes die in about 2300 rounds.

However, in other protocols, nodes start dying at about 850 rounds and leave certain black areas (hotspot problem) in the network, which are not accessible to the BS. As shown in Figure 8 , due to the maximum lifetime, the proposed scheme has maximum throughput as compared to the existing schemes.

The impact of the weight of each criterion is shown in Figure 9. The weights of each criterion vary from $0.25-0.50$. The simulation results show that assigning a value 0.5 to the weight $\left(\omega_{e}\right)$ which represents the residual energy criterion, results with a maximum lifetime.

That's why the nodes with high residual energy perform better as CHs. Furthermore, nodes with the higher number of neighbors consume more energy in data aggregation. Besides data aggregation, energy in node density parameter has the benefit of a large amount of data aggregation as well. It transmits data in a single unit and minimizes the overall network energy consumption. Therefore, parameters are considered for optimal $\mathrm{CH}$ selection in the proposed scheme, which reduces the average energy consumption of the network per round, as shown in Figure 10. 


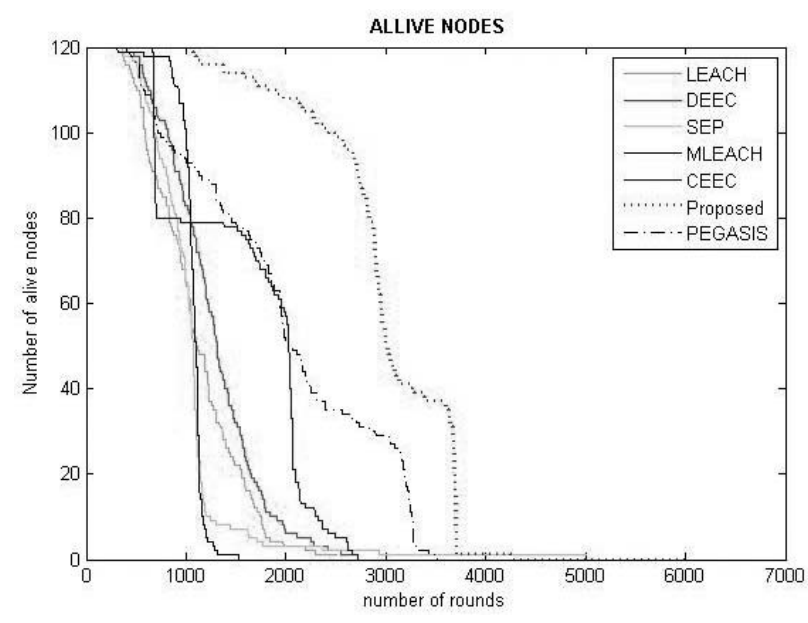

Figure 12. Network lifetime comparison of proposed model with state of the art routing protocols.

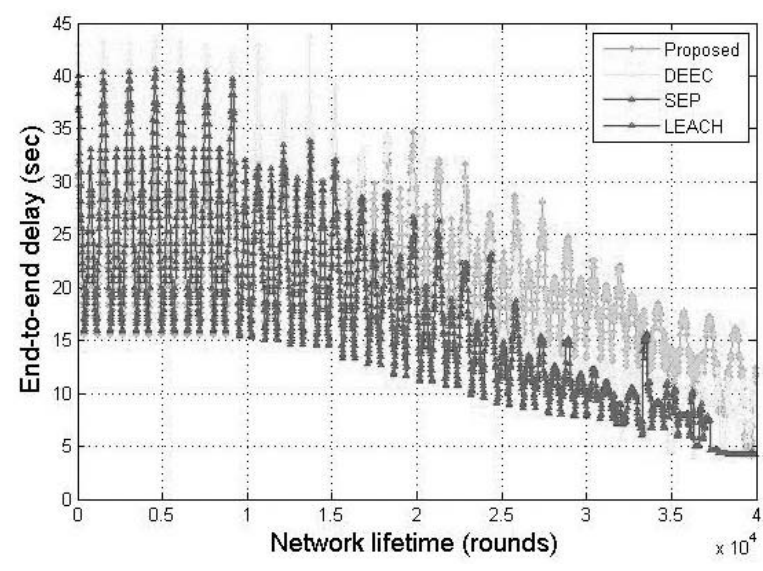

Figure 13. Comparison of network performance of proposed scheme with state of the art protocols.

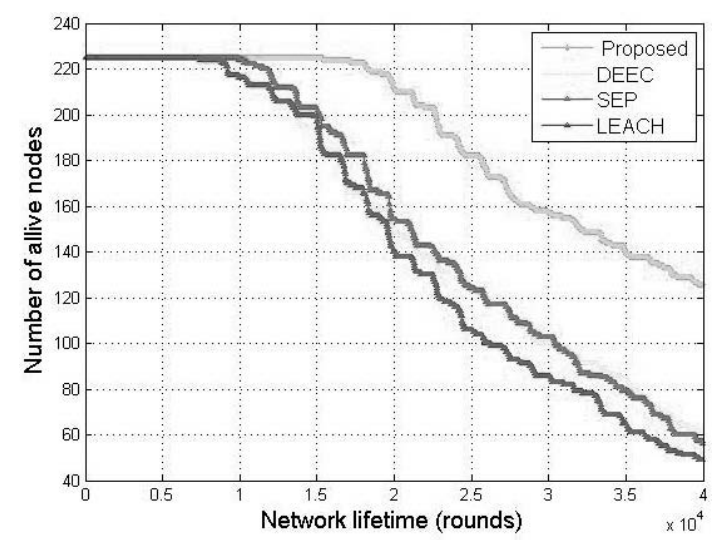

Figure 14. Network lifetime improvements of the proposed method as compared to well-known protocols.
Network lifetime of the compared routing protocols is also tested with different weights, as shown in Figure 11.

Consequently, the network lifetime of all the protocols increases. However, our proposed method still shows better lifetime as compared to the existing techniques.

Figure 12 indicates that the author has realistically compared the proposed protocol with the well-known state of the art routing protocols like LEACH, PEGASIS, DEEC, CEEC, M-LEACH, and SEP.

Results indicate that our proposed protocol outperforms all the existing compared protocols. In these simulation results, the proposed model has $70 \%$ better stability than CEEC, nearly $12 \%$ better network lifetime than PEGASIS; it should be noted that these protocols are the closest to our protocol in terms of performance.

Figures 13 and 14 clearly show the improvement of the proposed scheme regarding network performance and lifetime improvements compared to known state of the art protocols.

Hence, our proposed model has better network lifetime and stability period as compared to other existing protocols.

\section{Conclusion}

The paper proposed the OPEN routing path planning algorithm with energy balancing and optimized lifetime in WSN. The primary objective to focus on is the criteria of $\mathrm{CH}$ selection based on multiple parameters such as residual energy, the number of neighbors and the average distance of a sensor node from its neighbors. Along with these parameters, timer value concept is used to calculate. Any node with the least timer value within a cluster is selected as a $\mathrm{CH}$ for a particular round. The $\mathrm{CHs}$ near the BS has smaller cluster size as compared to the $\mathrm{CHs}$ located far from the BS. Therefore, the $\mathrm{CH}$ near the $\mathrm{BS}$, which acts as a leading node for all the other $\mathrm{CHs}$, has an equal load as compared to the CHs away from the BS. So, it balances the energy consumption among all the CHs. From the simulation results, it can be concluded that OPEN protocol performs better than the LEACH, DEEC, PEGASIS, CEEC and SEP protocols due to the optimal $\mathrm{CH}$ selection that results in better stability period, better data delivery and longer network lifetime. 


\section{References}

[1] W. R. Heinzelman et al., "Energy-Efficient Communication Protocol for Wireless Microsensor Networks", IIEEE 33rd Annual Proceedings of the Hawaii International Conference on System Sciences, Jan 4-7, 2000.

http://dx.doi.org/10.1109/HICSS.2000.926982

[2] G. Smaragdakis et al., "SEP: A Stable Election Protocol for Clustered Heterogeneous Wireless Sensor Networks", Second International Workshop on Sensor and Actor Network Protocols and Applications (SANPA 2004), Boston, Massachusetts, USA, 2004.

http://dx.doi.org/10.3923/jmcomm.2010.38.42

[3] A. Ahmad et al., "(ACH) 2: Routing Scheme to Maximize Lifetime and Throughput of Wireless Sensor Networks" Sensors Journal, IEEE, vol. 14, no.10, pp. 3516-3532, 2014. http://dx.doi.org/10.1109/JSEN.2014.2328613

[4] T. Shah et al., "VIKOR Based Distributed Clustering Scheme for Wireless Sensor Networks", in Frontiers of Information Technology (FIT), 2013 11th International Conference, pp. 253-258, 2013.

http://dx.doi.org/10.1109/FIT.2013.53

[5] D. Meenakshi and S.Kumar, "Energy Efficient Hierarchical Clustering Routing Protocol for Wireless Sensor Networks", in Computer Science and Information Technology, Networks and Communications, Springer Berlin Heidelberg, pp. 409-420, 2012.

http://dx.doi.org/10.1007/978-3-642-27299-8_43

[6] M. Aslam et al., "CEEC: Centralized Energy Efficient Clustering a New Routing Protocol for WSNs", in Sensor, Mesh and Ad Hoc Communications and Networks (SECON), 2012 9th Annual IEEE Communications Society Conference, pp. 103-105, 2012.

http://dx.doi.org/10.1109/SECON.2012.6275763

[7] V. Mhatre and C. Rosenberg, "Design Guidelines for Wireless Sensor Networks Communication, Clustering and Aggregation", Ad Hoc Networks, 2.1, pp. 45-63, 2004.

http://dx.doi.org/10.1016/S1570-8705(03)00047-7

[8] A. Wadaa et al., "On Providing Anonymity in Wireless Sensor Networks", Proceedings of the Parallel and Distributed Systems, Tenth International Conference, pp. 411, 2004.

http://dx.doi.org/10.1109/ICPADS.2004.56

[9] S. Olariu and I. Stojmenovic, "Design Guidelines for Maximizing Lifetime and Avoiding Energy Holes in Sensor Networks with Uniform Distribution and Uniform Reporting", INFOCOM, Barcelona, Spain, 2006.

http://dx.doi.org/10.1109/INFOCOM.2006.296
[10] O. Younis et al., "Node Clustering in Wireless Sensor Networks: Recent Developments and Deployment Challenges", IEEE Network, vol. 20, no. 3, pp. 20-25, 2006.

http://dx.doi.org/10.1109/MNET.2006.1637928

[11] B. P. Deosarkar et al., "Cluster Head Selection in Clustering Algorithms for Wireless Sensor Networks: A Survey", Computing, Communication and Networking IEEE International Conference, 2008.

http://dx.doi.org/10.1109/ICCCNET.2008.4787686

[12] W. B. Heinzelman et al., "An Application-Specific Protocol Architecture for Wireless Microsensor Networks", IEEE Transaction on Wireless Communications, vol. 1, no. 4, 2002. http://dx.doi.org/10.1109/TWC.2002.804190

[13] Y. Li et al., "The Algorithm of Cluster Head Multi-Hops Based on LEACH", 17th Computer Engineering and Design, vol. 17, no. 33, 2007. (Chinese Journal)

[14] Y. C. Jia et al., "Hierarchical Clustering Routing Scheme Based on LEACH in Wireless Sensor Networks", Computer Engineering, vol. 35, no. 11, pp. 74-76, 2009.

http://www.ecice06.com/EN/Y2009/V35/I11/74

[15] D. Kumar et al., "EEHC: Energy Efficient Heterogeneous Clustered Scheme for Wireless Sensor Networks", Computer Communications, vol. 32, no 4, pp. 662-667, 2009.

http://dx.doi.org/10.1016/j.comcom.2008.11.025

[16] L. Qing et al., "Design of a Distributed Energy-Efficient Clustering Agorithm for Heterogeneous Wireless Sensor Networks", Computer Communications, vol. 29, no. 12, pp. 2230-2237, 2006.

http://dx.doi.org/10.1016/j.comcom.2006.02.017

[17] L. Ming et al., "EADEEG: An Energy-Aware data Gathering Protocol for Wireless Sensor Networks", Journal of Software, vol. 18, no. 5, pp. 1092-1109, 2007.

http://dx.doi.org/10.1360/jos181092

[18] Z. Xinlian et al., "BPEC: An Energy-aware Distributed Clustering Algorithm in WSNs", Journal of Computer Research and Development, vol. 46, no. 5, pp. 723-730, 2009. (Chinese Journal)

[19] O. Younis and S. Fahmy, "HEED: a Hybrid, Energy Efficient Distributed Clustering Approach for ad-hoc Sensor Networks", IEEE Transactions on Mobile Computing, vol. 3, no. 4, pp. 366-379, 2004. http://dx.doi.org/10.1109/TMC.2004.41

[20] C. F. Chiasserini et al., "Energy Efficient Design of Wireless ad hoc Networks", in NETWORKING 2002: Networking Technologies, Services, and Protocols, Performance of Computer and Communication Networks, Mobile and Wireless Communications, Springer, Berlin Heidelberg, 
pp. 376-386, 2002.

http://dx.doi.org/10.1007/3-540-47906-6_30

[21] S. Lindsey and C. S. Raghavenda, "PEGASIS: Power Efficient Gathering in Sensor Information Systems", in IEEE, Aerospace Conference, Big Sky, Montana, March 2002.

http://dx.doi.org/10.1109/AERO.2002.1035242

[22] N. Javaid et al., "MCEEC: Multi-Hop Centralized Energy Efficient Clustering Routing Protocol for WSNs", 48th IEEE International Conference on Communications, ICC, Sydney, Australia, 2014. http://dx.doi.org/10.1109/ICC.2014.6883581

[23] A. Iqbal et al., "Advanced LEACH: A Static Clustering-Based Heterogeneous Routing Protocol for WSNs", arXiv preprint arXiv, vol. 1306, no. 1146, 2013.

[24] S. Stanislava and W. B. Heinzelman, "Prolonging the Lifetime of Wireless Sensor Networks via Unequal Clustering", in Parallel and Distributed Processing Symposium, Proceedings, 19th IEEE International, pp. 8, 2005. http://dx.doi.org/10.1109/IPDPS.2005.365

[25] C. Li et al., "An Energy-Efficient Unequal Clustering Mechanism for Wireless Sensor Networks", IEEE International Conference on Mobile Adhoc and Sensor Systems, 2005. http://dx.doi.org/10.1109/MAHSS.2005.1542849

[26] G. Zanca et al., "Experimental Comparison of RSSI-Based Localization Algorithms for Indoor Wireless Sensor Networks", in The ACM Workshop on Real-World Wireless Sensor Networks, pp. 1-5, 2008.

http://dx.doi.org/10.1145/1435473.1435475

[27] I. Demirkol et al., "MAC Protocols for Wireless Sensor Networks: a Survey", IEEE Communications Magazine, vol. 44, no. 4, pp. 115-121, 2006. http://dx.doi.org/10.1109/MCOM.2006.1632658

[28] G. Hossein Ekbatani Fard et al., "An Adaptive Cross-Layer Multichannel QoS-MAC Protocol for Cluster-Based Wireless Multimedia Sensor Networks", IEEE Xplore, Conference paper, September 2009.

http://dx.doi.org/10.1109/ICUMT.2009.5345446

[29] N. Sabena et al., "Dynamic Duty Cycle and Adaptive Contention Wwindow Based QoSMAC Protocol for Wireless Multimedia Sensor Networks", Computer Networks, vol. 52, pp. 2532-2542, 2008. http://dx.doi.org/10.1016/j.comnet.2008.05.009
Contact addresses:

Syed Bilal Hussain Shah

School of Information and Communication Engineering Faculty of Electronic Information and Electrical Engineering Dalian University of Technology Dalian, China e-mail: bilalshah@mail.dlut.edu.cn

Zhe Chen

School of Information and Communication Engineering Faculty of Electronic Information and Electrical Engineering Dalian University of Technology Dalian, China e-mail: zhechen@dlut.edu.cn

Fuliang Yin

School of Information and Communication Engineering Faculty of Electronic Information and Electrical Engineering Dalian University of Technology Dalian, China e-mail: flyin@dlut.edu.cn

Syed Bilal Hussain Shah is currently a PhD scholar at the School of Communication and Information Engineering, Dalian University of Technology (DUT) P. R. China. He got the B.S degree in computer sciences (2007) from the Department of Computer Sciences, University of Peshawar, Pakistan. He joined Bahria University Islamabad, Pakistan where he earned the Masters degree in telecommunication and networking (2009). He worked as a Lecturer at the Department of Computer Sciences University of Peshawar $(2010$ - 2012) Pakistan. His main research interests include energy efficient routing in wireless networks, distributed and centralized clustering in WSN, game theory in Wireless Sensor Networks (WSN), game theory in WSN, and Internet of Things.

ZHE CHEN received his B.S degree in electronic engineering, the M.S degree in signal and information processing, and the $\mathrm{PhD}$ degree in signal and information processing from Dalian university of Technology (DUT), Dalian, China, in 1996, 1999 and 2003 respectively. He joined the Department of Electronics Engineering, DUT, as a lecturer in 2002, and became an Associate Professor in 2006. His research interests include speech processing, image processing and wideband wireless communication.

Fuliang YIN was born in Fushun city, Liaoning province, China, in 1962. He received his B.S degree in electronic engineering and M.S. degree in communications and electronic systems from Dalian University of Technology (DUT), Dalian, China, in 1984 and 1987, respectively. He joined the Department of Electronic Engineering, DUT, as a lecturer in 1987 and became an Associate Professor in 1991. He has been a Professor at DUT since 1994, and the Dean of the School of Electronic and Information Engineering of DUT from 2000 to 2009. His research interests include digital signal processing, speech processing, and image processing and broadband wireless communication. 